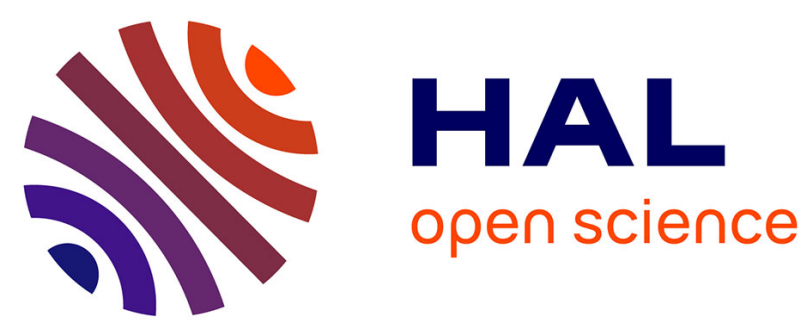

\title{
Nîmes et les Volques Arécomiques au Ier siècle avant J.-C.
}

\author{
Michel Christol, Christian Goudineau
}

\section{To cite this version:}

Michel Christol, Christian Goudineau. Nîmes et les Volques Arécomiques au Ier siècle avant J.C.. Gallia - Fouilles et monuments archéologiques en France métropolitaine, 1988, 45, pp.87-103. 10.3406/galia.1987.2881 . hal-01921053

\section{HAL Id: hal-01921053 \\ https://hal.science/hal-01921053}

Submitted on 14 Jan 2020

HAL is a multi-disciplinary open access archive for the deposit and dissemination of scientific research documents, whether they are published or not. The documents may come from teaching and research institutions in France or abroad, or from public or private research centers.
L'archive ouverte pluridisciplinaire HAL, est destinée au dépôt et à la diffusion de documents scientifiques de niveau recherche, publiés ou non, émanant des établissements d'enseignement et de recherche français ou étrangers, des laboratoires publics ou privés.

\section{(ㅇ)(1) $\$$}

Distributed under a Creative Commons Attribution - NonCommercial - NoDerivatives| 4.0 


\title{
NIMES ET LES VOLQUES ARÉCOMIQUES AU I ${ }^{\text {er }}$ SIÈCLE AVANT J.-C.
}

\author{
par Michel CHRISTOL et Christian GOUDINEAU
}

En 1976, l'un de nous publiait un article consacré au statut de Nimes et des Volques Arécomiques qui, reprenant les textes de César (B.C., I, 35) et de Strabon (IV, 1, 12), en proposait une relecture dont on peut ainsi résumer les conclusions : 1. c'était Pompée (et non César) qui avait "concédé" à Marseille agros Volcarum Arecomicorum et Helviorum, César lui "attribuant" ultérieurement des territoires (ou l'équivalent : le mot latin a disparu de la tradition manuscrite) en Gaule intérieure; 2. à Nimes, colonie de droit latin, avaient été "attribuées» vingt-quatre communautés arécomiques (les oppida ignobilia de Pline, III, 37) qui, cependant, jouissaient également du droit latin; ces mesures avaient été prises à l'époque césarienne ou au début de l'époque triumvirale ${ }^{1}$.

Bien que ces propositions aient été en général retenues, elles laissaient un sentiment d'insatisfaction, non au plan de la philologie mais pour l'explication historique. Pourquoi César ou Lépide avaient-ils attribué à Nimes vingt-quatre oppida arécomiques? Comment expliquer l'extraordinaire parure monumentale de Nimes à l'époque augustéenne (le don par Auguste des murs et des portes, les transformations de la Tour Magne et du quartier de la Fontaine, l'édification de la Maison Carrée, etc.)? Comment rendre compte du monnayage "au crocodile» qui allait diffuser dans tout l'Occident le nom de la COL NEM "patronnée» en quelque sorte par Auguste et Agrippa? Pourquoi autant d'inscriptions? Pourquoi Nimes, à haute époque, devança-telle toutes les civitates de Gaule par le nombre des

1 Chr. Goudineau, Le statut de Nimes et des Volques Arécomiques, Revue Archéologique de Narbonnaise (R.A.N.), 9, 1976, p. 105-114. élévations (chevaliers et sénateurs)? Pourquoi la titulature COL.AVG(usta)? On n'en finirait pas.

Pour ouvrir de nouveau le dossier, il fallait ajouter à l'étude des textes l'épigraphie, la numismatique et de récentes découvertes ou interprétations archéologiques. Vaste programme. Cependant, plutôt que de donner à nos recherches la forme d'un livre, avec ce qu'il aurait eu de faussement définitif, nous avons préféré les proposer sous forme concise, quitte à ne pas argumenter dans le détail : les travaux sont nombreux en terre arécomique, et beaucoup de chercheurs pourront alimenter le débat ${ }^{2}$.

\section{L'ORGANISATION POMPEIENNE}

L'histoire de Nimes et des Volques Arécomiques, au $\mathrm{I}^{\mathrm{er}} \mathrm{s}$. av. J.-C., est généralement écrite en fonction de Marseille. Celle-ci aurait exercé une domination qui ne cessa qu'avec sa capitulation

2 Une mise au point a èté récemment présentée par M. Py, J.-L. Fiches et P. Garmy dans les deux chapitres "Nimes protohistorique» et "Nimes gallo-romaine" de l'II istoire de Nimes, Edisud, 1982, avec une bibliographie qu'on peut augmenter à l'aide des nombreuses références qui accompagnent les articles de D. Roman, particulièrement La fondation de la colonie de Nimes : problèmes de chronologie, Bulletin de l'Ecole Antique de Nimes, 14, 1979, p. 99-104 et Apollon, Auguste et Nimes, R.A.N., 14, 1981, p. 207-214. Les problèmes que nous évoquons devront être complètés par l'étude des cadastrations, actuellement en cours : Cf. J.-L. Fiches et J. Soyer, Occupation du sol et cadastres antiques : l'exemple de la carte de Nimes, Cadastres et espace rural, Paris, 1983, p. 259-274. 
a
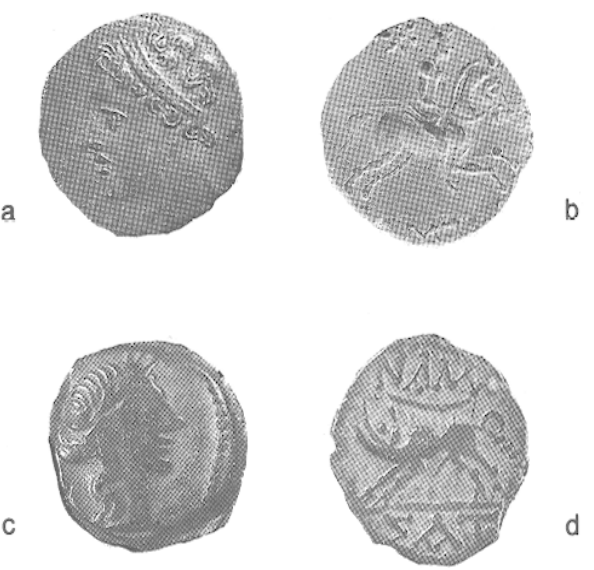

e
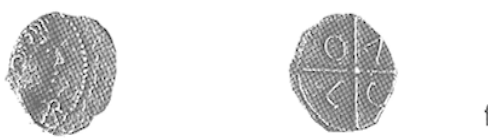

g
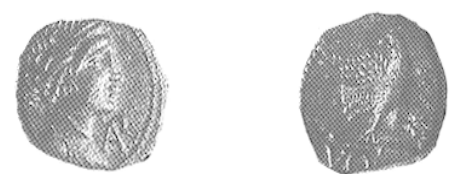

h

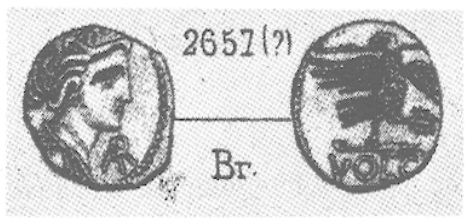

i
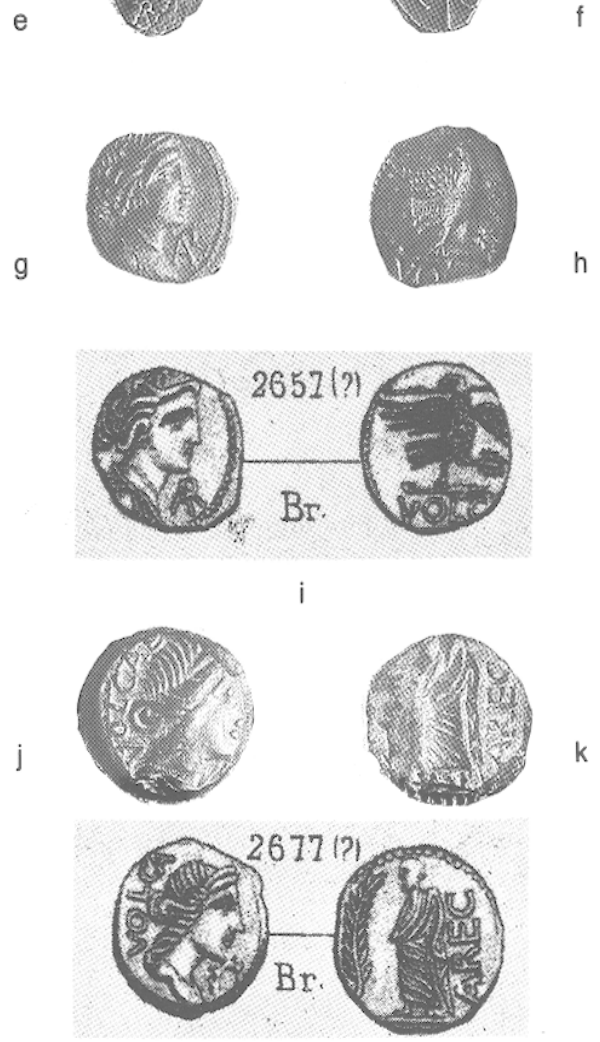

Fig. 1 - a, b, émission NEMAr; c, d, émission NAMALAT; $\mathrm{e}, \mathrm{f}$, émission d'argent VOLC AR; $\mathrm{g}, \mathrm{h}$, i, petit bronze à l'aigle; j, k, l, bronze dit "au dèmos».

devant César. Une bonne illustration de la dépendance puis de la libération serait offerte par le monnayage : à la drachme et au petit bronze portant les légendes grecques NEMAr et NAMA raient succédé les émissions à légende latine VOLC AR (ou AREG) montrant, notamment, un personnage en toge devant une palme, symbole de la liberté retrouvée et du droit latin accordé en 49 ou un peu plus $\operatorname{tard}^{3}$ (fig. 1).

Revenons à César B.C., I, 35. Avec laconisme, le texte rappelle en quelques mots les bienfaits de Pompéc et ccux de César à l'égard des Marseillais : "(Pompée) leur a concédé officiellement des terres des Volques Arécomiques et des Helviens, (luimême) leur a attribué des (...) de Gaule qu'il venait de vaincre, augmentant ainsi leurs revenus». La lacune restituée peut se combler, par exemple, par partes.

La démarche n'a rien d'inhabituel, qui consiste à donner à des communautés - ou à des particuliers - soit des terres soit des revenus (des rentes foncières) à l'extérieur du territoire de leur propre cité. Les exemples ne manquent pas pour la période qui nous intéresse ${ }^{4}$. Les "concessions" pompéiennes consistèrent, au moins en partie, en dons de terres puisqu'il fallut recourir à la force armée pour réaliser les expropriations (Pro Fonteio, 13, 14, 26). Les

3 Longue tradition sur ce petit bronze dit «au dèmos». En dernier lieu, J.-L. Fiches et J.-Cl. Richard, L'émission monétaire d'Ambrussum, R.A.N., 18, 1985, p. 387 : "le personnage en toge du revers des petits bronzes (...) pourrait même symboliser l'obtention du droit latin". Mais nul n'a jamais fourni de comparaison numismatique allant en ce sens. Sur le monnayage de Nimes, la seule étude de synthèse demeure celle de J.-B. Giard, Le monnayage antique de Nimes, Bulletin de l'École Antique de Nimes, 6-7, 1971-1972, p. 47-60, tributaire d'hypothèses historiques.

4 Entre autres exemples, rappelons les cas clairement exposés par Gicḱron, Fam., XIII, 7, 1 et 11, I, concernant Atella et Arpinum, municipes auxquels furent attribuées des rentes foncières sur des territoires gaulois : les expressions utilisées (agro vectigali, vectigalia) sont semblables à celles de César. Plus tard (en 38), Velleius Paterculus II, 81 (confirmé par Dion Cassius, XLIX, 14, 5) signale qu'à Capoue, en échange d'une extension du domaine colonial, furent données «dans l'île de Crète des terres plus fertiles dont le revenu était de 1200 mille sesterces - et on lui promit l'eau (la future Aqua Julia)». Une contribution récente de J.-M. Bertrand, Le statut du territoire attribué dans le monde grec et romain, Sociétés urbaines, sociétés rurales dans l'Asie Mineure et la Syrie hellénisliques et romaines, Strasbourg, 1987, p. 95-106, propose un réexamen de ces questions d'attribution, contestant de fond en comble la demarche d'U. LAFFi, Adtributio e contributio, problemi del sistema amministrativo dello stato romano, Pise, 1966 : "Le travail d'U. Laffi révèle (...) qu'il a construit lui-même, au fur et à mesure qu'il croyait l'étudier, le 'senso tecnico' d'un mot qui n'existait pas dans l'emploi qu'il feint de décrire. Il suffit en effet de jeter un œil sur le Thesaurus pour s'apercevoir qu'il n'y a pas d'occurrence du mot altributio qui puisse justifier le sens qu'il lui découvre». Sa définition est simple : «l'attribution est tout naturellement le prolongement d'une conquête, le vainqueur donnant à qui l'a aidé dans la guerre tout ou partie du territoire qui vient de lui revenir». La démonstration paraît convaincante. 
bienfaits césariens pouvaient être de même nature, si on en juge par ce qu'il fit en faveur de deux nobles allobroges ${ }^{5}$. Il n'est donc pas utile d'insister sur ce procédé sauf pour rappeler qu'il n'implique pas nécessairement la subordination juridique d'une communauté à une autre ${ }^{6}$.

Ce qui nous intéresse ici, c'est que, évoquant l'action de Pompée, César cite nommément les Volques Arécomiques (et les Helviens). Le mot publice ("officiellement", "au nom de l'État») implique un acte juridique que Pompée a accompli comme représentant du Sénat et du peuple Romain? Divers indices accumulés depuis une suggestion de Badian $^{8}$ laisseraient supposer que ces concessions concordèrent avec la première organisation juridique de la provincia, autrement dit avec la rédaction de la lex provinciae, laquelle aurait nommé les peuples (pour la première fois, apparaissent les Arécomiques) et précisé les contraintes pesant sur eux ${ }^{9}$. On note que, peu après, le Pro Fonteio ne met dans la bouche des plaignants (Volques ${ }^{10}$ et Allobroges) aucune récrimination quant à leur statut: leurs plaintes sont suscitées par des abus.

5 B.C., III, 49 : "Il leur avait donné dans leur patrie les plus hautes magistratures, à titre exceptionnel il les avait fait entrer au sénat (de Rome) et leur avait attribué (tribuerat) des terres prises en Gaule sur les ennemis et de grandes récompenses d'argent". Sur des accroissements territoriaux ou (aussi bien) des attributions de terres, cf. par exemple Dion, XLIII, 39. Nombreux autres exemples dans Bertrand, ibidem.

6 Arpinum et Atella n'exercent aucune domination sur les peuples dont elles reçoivent des vectigalia. Il faut ici distinguer entre des usages anciens tenant du droit de guerre et des mécanismes plus complexes relevant de compensations ou de faveurs accordées à des cités ou à des individus.

7 Le Pro Fonteio ne distingue pas clairement les actes accomplis par Pompée ou par Fonteius en vertu des pouvoirs qui leur avaient été délégués (par exemple, l'habilitation reçue par Pompée, sur avis du Sénat et aux termes de la lex Gellia Cornelia, d'accorder la civilas à des personnes physiques) ou pour exécuter des décisions du Sénat. Pro $F$., 14 : ex agris ex Cn. Pompei decreto decedere sunt coacti semble plaider en faveur de la première hypothèse.

8 E. Badian, Notes on Provincia Gallia in the late Republic, Mélanges Piganiol, II, 1966, p. 901-918.

9 C'est la conclusion à laquelle étaient arrivès indépendamment Ch. EBEL, Transalpine Gaul, the emergence of a Roman province, Leyde, 1976, p. 74-102 et Chr. Goudineau, dans $\mathrm{CI}$. Nicol.ET, Rome et la conquête du monde méditerranéen, 2, Paris, 1978, p. 692. Notons que le gentilice Pompeius est bien représenté chez les Arécomiques : environ $3 \%$. Sur la diffusion des gentilices, cf., entre autres, Y. Burnand, Domitii Aquenses, Paris, 1975, p. 226-228.

10 On aimerait évidemment savoir si les Volques cités par le Pro $F$., 26 avec les Allobroges sont Arécomiques ou Tectosages. Si les allusions aux dépossessions de terres concernent les premiers, l'affaire de la voie domitienne peut
S'il en est bien ainsi, c'est aux années 75 environ qu'il faut faire remonter de la part de Rome la reconnaissance (ou la définition) officielle, juridique et territoriale d'une entité des Volques Arécomiques $^{11}$. La province est organisée : les Arécomiques en constituent une part, même si des dispositions prévoient la remise aux Massaliotes de terres ou de vectigalia. On peut écarter les hypothèses qui placeraient le territoire sous la domination de Marseille : aucun argument ne les fonde (au contraire, il s'agit, pour la première fois, de codifier en termes précis ce qui est octroyé à Marseille dans un territoire désormais pris en charge par Rome) et, par ailleurs, la comparaison avec les Helviens est éclairante. Ces derniers sont cités par César au même titre que les Arécomiques comme ayant fait l'objet de concessions d'agros par Pompée aux Massaliotes. Or, dans le Bellum Gallicum, les Helviens apparaissent comme un peuple jouissant d'un territoire et d'institutions stables, dirigé par un princeps; deux fils de l'un de ces principes accompagnèrent César ${ }^{12}$.

Il faut donc distinguer clairement la reconnaissance des Arécomiques comme entité constituante de la provincia et le fait, annexe, que, comme les Helviens, ils furent frappés par les privilèges territoriaux et financiers accordés à Marseille ${ }^{13}$.

viser les deux (encore que Narbonne soit placée par Strabon IV , 1,12 chez les Arécomiques) et la taxe sur le vin plutôt les Tectosages.

11 Si le triennium de Fonteius se place entre 74 et 72 (T.R.S. Broughton, The magistrates of the Roman Republic, N.Y., 1950-1960, II, p. 97, 104, 109; E. BADIAN, ibid., p. $911 s q$. mais d'autres préfèrent 76-74) et s'il met en application la nouvelle lex provinciae, celle-ci se date entre le départ de Pompée pour l'Espagne et, au plus tard, 72.

12 C. Valerius Procillus et son frère $\mathrm{C}$. Valerius Donnotaurus étaient les fils du princeps des Helviens C. Valerius Caburus; le premier est désigné par César comme familiaris suus (B.G., I, 19). Sur les Helviens à l'époque républicaine, $\mathrm{R}$. Lauxerois, Le Bas-Vivarais à l'époque romaine, suppl. 9 à la R.A.N., p. 70-75.

13 Reste le problème du monnayage VOLC AREC. Notons d'abord que cette émission, qui comporte une pièce d'argent et deux petits bronzes, offre un élément chronologique relevé par GIARD, loc. cil. : le petit bronze à l'aigle reprend le revers d'un denier de Pomponius Rufus; M. Crawford, dans la nouvelle édition de Roman Republican Coinage, Cambridge, 1983, p. 410, no 398, le date de 73 avant J.-C. La description précise de ce revers laisse une incertitude : l'aigle, qui tient une couronne dans sa serre gauche, est-il perché sur un sceptre ou sur une palme? D'autre part, le personnage en toge, plutôt que d'évoquer le droit latin accordé aux Arécomiques, pourrait faire partie des personnages ou des symboles liés à la source, nombreux dans les monnayages de Nimes (infra). Or, l'homme en toge se trouve dans le cycle de Salus ou de Valetudo: cf., par exemple, A. Garcia y Bellido, Esculturas 


\section{L'EEUVRE CÉSARIENNE}

Bien que César n'en dise mot, c'est vraisemblablement à l'issue de la guerre des Gaules ou vers 4948 qu'il accorda le droit latin aux communautés de Transalpine. Ces mesures coïncidèrent-elles avec le démembrement du territoire massaliote? Rien ne le prouve et, répétons-le, la possession ou l'attribution de telle ou telle terre n'avait pas de rapport direct avec le statut juridique de la communauté. Une telle concordance serait cependant satisfaisante pour l'esprit. De ces communautés de droit latin, oppida latina, un état fut dressé, annexé à la lex provinciae ou intégré à celle-ci. Le texte de Pline, III, 31-37, nous en restitue partiellement la teneur.

Partiellement car ce n'est pas cet état de la formula qui servil de source directe au Naturaliste. La liste des oppida latina situés in mediterraneo comprend trente noms, présentés par ordre alphabétique (selon la digestio in litteras signalée en III, 46). L'un d'entre eux, Augusta Tricastinorum, donne un terminus post quem obligé à partir de 27 avant J.-C. C'est donc à une refonte de la lex provinciae que se réfère Pline, une refonte quasi définitive puisqu'on put enregistrer quelques aménagements ultérieurs sans avoir à la recomposer (voir infra). Tout laisse supposer que cette rédaction fut accomplie sous la supervision de l'empereur, probablement dès son séjour à Narbonne de 27 avant J.-C. (Liv., Per., 134) au cours duquel il procéda au recensement et à l'organisation des Gaules (Dion Cassius, LIII, 22, 5, faisant allusion au "bios» et à la "politeia» des habitants). Cependant, aux dires mêmes de Dion, le principal souci du prince était de mettre en ordre les régions conquises par César : les décisions relatives à la Narbonnaise ne durent être que des aménagements ou le parachèvement d'une organisation dont

romanas de España y Portugal, Madrid, 1949, nº 493. Enfin, la chronologie de ce monnayage n'est pas fermement établie par les trouvailles archéologiques : selon M. PY, L'oppidum des Castels à Nages, suppl. 35 à Gallia, 1978, p. 309, "il semble succéder à la monnaie au sanglier vers les années $70 \%$. Dans une étude encore inédite rassemblant toutes les données à sa disposition, M. Py accorde une antériorité aux monnaies VOLC AREC sur celles à légende NEM COL, et il les place dans "le deuxième tiers du I $^{\text {er }}$ s.». J.-L. Fiches et J.Cl. Richard (cf. note 3 ) admettent la chronologie relative mais suivent une interprétation historique dont J.-B. Giard écrivait (p. 50) : "est-ce bien certain?". Dans un tel contexte, nous suggérons de nous en tenir au seul élément incontestable : la datation du denier de Pomponius Rufus. La date de 73 représente un terminus posl quem mais rien n'oblige à supposer un long dèlai. les grandes lignes avaient été mises en place auparavant, c'est-à-dire au temps de César.

Il faut donc chercher à retrouver, à l'intérieur de la source de Pline, les traces de l'organisation césarienne plus ancienne. Même si l'ordre d'exposition qu'il suit, mi-géographique mi-administratif, ne facilite pas la tâche et même si des incertitudes chronologiques demeurent, on peut proposer comme oppida latina césariens :

- sur le littoral, Ruscino (peu après, Antipolis : Strabon, IV, 1, 9);

- à l'intérieur des terres : trente noms depuis Aix jusqu'aux duo capita des Voconces, Vaison et Luc;

- quarante-trois oppida ignobilia sur lesquels nous reviendrons mais dont on peut dire qu'à l'époque césarienne ils constituaient, à l'égal des précédents, des communautés autonomes pourvues du droit latin.

En laissant de côté les colonies romaines (coloniae) dont certaines ont pu jouir du statut d'oppida latina avant leur déduction ou leur élévation au droit romain, et sans tenir compte d'éventuels remaniements limités, on se trouve devant un total de trente et un oppida lalina nommément cités et de quarantetrois oppida ignobilia, soit en tout soixante-quatorze oppida latina. Que représentent-ils? L'ensemble des communautés de la provincia dotées d'une autonomie minimale qu'on ne sait évaluer avec précision, mais qui trouve des illustrations dans certains monnayages, voire dans l'inscription gallo-grecque

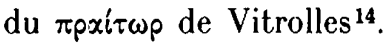

Dans le cadre de l'unité "Volques Arécomiques", la formula césarienne énumérait vingt-cinq communautés : Nimes plus vingt-quatre oppida latina dont Strabon et Pline ne citent pas les noms pour des raisons que nous examinerons infra.

L'étape suivante fut, selon nous, la déduction à Nimes d'une colonie de droit latin en 45 ou 44 . Les indices résident moins dans le monnayage NEM COL (datable des environs de 40-30, on y reviendra) que dans l'étude archéo-topographique publiée naguère par J. Benoit et qui n'a suscité que peu d'écho ${ }^{15}$.

14 En dernier lieu, M. Lrjeune, R.I.G., I, suppl. 45 à Gallia, 1985, p. 123-125 : le personnage pourrait être magistrat. des "Soma (...)" représentant un oppidum ultérieurement devenu ignobile. Des homologues ont pu frapper monnaie : L. Chaвot, Ch. Kurtz, Cahiers Numismatiques, 54, 1977, p. $100-103$.

15 J. Benort, Nimes : études sur l'urbanisme antique, Bulletin de l'École Antique de Nimes, 16, 1981, p. 69-90. 

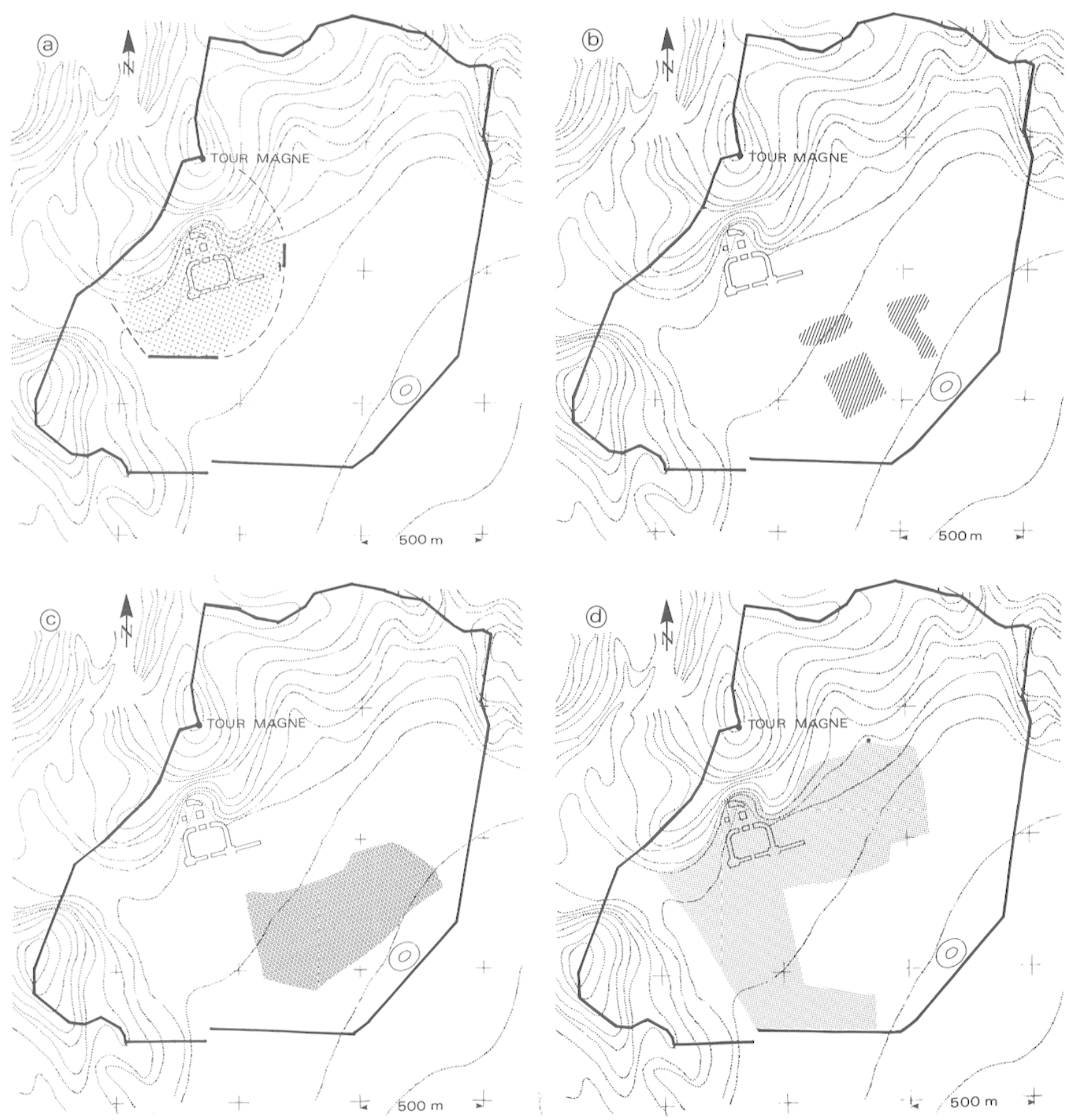

Fig. 2 - Les phases successives de l'urbanisation de Nimes, d'après J. Benoit : a, l'oppidum; b, les traces de la "ville verte"; c, la "ville violette»; d, la "ville rouge" (augustéenne).

Analysant le cadastre moderne de Nimes (fig. 2), J. Benoit a montré qu'il conservait les traces de carroyages antiques, parmi lesquels l'un correspondait à la ville augustéenne, telle qu'on en connaît les orientations (notamment par la Maison Carrée). Trois autres lui étaient antérieurs. Le plus ancien recouvre les pentes du Mont-Cavalier et ses environs, c'est-à-dire l'oppidum protohistorique. Il en reste deux. Le premier, situé dans le centre-ouest de la ville actuelle, occupe au minimum trois hectares; il est fondé sur le pied de $29,6 \mathrm{~cm}$ et organise des îlots de $96 \times 32 \mathrm{~m}$ et des rues de $4 \mathrm{~m}$ ("ville verte»); le second en représente l'extension ("ville violette»).

De quoi s'agit-il? Il n'existe pas de multiples possibilités : si l'on écarte l'hypothèse peu vraisemblable du camp romain, il ne reste que celle d'une fondation coloniale, c'est-à-dire d'une déduction. Une déduction qui serait donc pré-augustéenne. 
C'est là que nous retrouvons (une fois de plus!) la fameuse phrase de Suétone (Tib., 4) qui nous indique que le père du futur empereur Tibère, Tiberius Nero, fut envoyé en Gaule par César "pour y déduire des colonies parmi lesquelles Narbonne et Arles». Si la date de la mission est bien établie (en 45 , peut-être vers la fin de l'année ${ }^{16}$ ), sa nature fait problème: quelles autres colonies furent déduites ${ }^{17}$ ? A l'aide d'arguments variés, on a proposé d'ajouter (à Narbonne et à Arles) Orange, Béziers, voire Fréjus, c'est-à-dire des colonies de droit romain. L'étude de la fondation de Lyon nous a suggéré que Suetone pouvait faire allusion à des déductions de droit latin dont il ne cite pas le nom pour une raison simple : l'usage instauré sous l'empire réserve le titre des colonies à celles qui jouissent du droit romain. Parmi ces déductions latines, nous avons cité Vienne (déduction avortée) ${ }^{18}$. L'étude de J. Benoit recommande d'ajouter Nimes.

Un autre texte de Suétone (Tib., 13), qui concerne les Nimois, trouverait là une explication plausible. En 6 avant J.-C., Tibère, qui avait entamé une carrière brillante émaillée de triomphes, «résolut de se retirer" pour des motifs qui demeurent mystérieux (dégoût de sa femme? noble détermination de laisser le champ libre aux enfants d'Agrippa, Caius et Lucius, adoptés par Auguste, âgés de 14 et 11 ans?). Peu importe : il s'installa à Rhodes, où Auguste (dicil Suétone) le pria de rester. Il se mit à vivre à la grecque: "l'équitation, l'escrime, qui étaient ses exercices habituels, il les abandonna; il remisa même le costume de ses ancêtres pour adopter le manteau et les sandales grecques. C'est dans un tel état qu'il persévéra près de deux ans, s'attirant de jour en jour de plus en plus de mépris et de haine au point que ses représentations et ses statues furent renversées par les Nimois».

16 Mise au point vraisemblable par M. GaYraud, Narbonne antique, suppl. 8 à la R.A.N., p. 178-180. Cf. aussi J. Gascou, Quand la colonie de Forum Julii fut-elle fondée?, Latomus, 41, 1982, p. 132-134.

17 Bibliographie gigantesque, rappelée dans Chr. GouDineau, Les fouilles de la Maison au Dauphin, suppl. 37 à Gallia, 1979, p. 265 et suiv. Pour les auteurs comme Mommsen ou Jullian, la réponse était simple : les colonies romaines de Transalpine remontaient à cette date. Depuis lors, le débat a été complexe, avec des repentirs : par exemple, M. Clavel a "remonté» Béziers de l'époque triumvirale à l'époque césarienne, et l'un de nous (C.G.), suivant P. A. Brunt, en a fait autant avant de s'apercevoir que la voie était sans issue.

18 Chr. Goudineau, Note sur la fondation de Lyon, Gallia, 44, 1, 1986, p. 171-173.
Que viennent faire les Nimois dans cette galère? Une prise de position aussi violente dans une querelle de succession qui, au demeurant, semblait réglée paraît peu vraisemblable ${ }^{19}$; voir dans cette émeute le symptôme d'un anti-hellénisme traduisant d'anciennes rancœurs à l'égard de Marseille ne convainc pas davantage. En revanche, si l'on admet que Tibère, fils de Tiberius Nero fondateur de la colonie, en était devenu le patron, son exil et sa déchẻance pouvaient déclencher indignation et animosité ${ }^{20}$.

Récapitulons : la lex provinciae des années 75 définit une unité des Volques Arécomiques, même si ceux-ci (comme on le voit par l'attribution ultérieure du droit latin) sont répartis en de nombreuses communautés, peut-être vingt-cinq si l'on se réfère aux oppida latina; elle précise d'autre part les contraintes qui pèsent sur eux : non seulement celles que Rome impose aux peuples sujets mais aussi des dépossessions au bénéfice des Massaliotes. Vers 5248, César accorde le droit latin à la provincia et donc aux vingt-cinq communautés arécomiques, tandis que la capitulation de Massalia entraîne la suppression de ses privilèges territoriaux. Enfin, en $\mathbf{4 5}$ (ou à peu près), Tibérius Nero déduit une colonie de droit latin à Nimes.

19 En dernier lieu, R. Амy, P. Gros, La Maison Carrée de Nimes, suppl. 38 à Gallia, 1979, p. 193-194, et P. Gros, L'augusteum de Nimes, R.A.N., 17, 1984, p. 134, n. 90, selon lequel cet acte manifeste "l'étonnant conditionnement d'une population très attachée au clan d'Agrippa et, par voie de conséquence, hostile à celui de Livie". Mais, à cette date, les fils d'Agrippa étaient héritiers déclarés et Tibère éliminé : pourquoi tirer sur une ambulance? Une remarque : cette interprétation d'une "lutte de clans" conduit P. Gros (Maison Carrée, p. 193) à dater la dédicace du temple avant l'adoption de Tibère : «il est peu vraisemblable qu'une cité provinciale, si attachée qu'elle fût au clan d'Agrippa, ait après cela pu ou osé manifester une dévotion publique à l'égard des Caesares" et, donc, la mention PRINCIPIBVS IVVENTVTIS (qui manifesterait *la foi confuse en unc survic divine et le désespoir latent d'une colonie") se placerait au plus tard dans les derniers mois de 4 ou les premiers de 5 après J.-C. Mais, à cette date, Auguste est bien en vie : honorer ses fils adoptifs défunts ne comporte ni scandale ni risque. Ce terminus ante quem de l'inscription de la Maison Carrée (qui n'est pas sans conséquence pour les datations de l'architecture gallo-romaine) est-il fondé?

20 Ces patronages héréditaires sont bien connus : en dernier lieu, L. KePpIE, Colonisation and veteran setllement in Italy, British School at Rome, 1983, p. 97 et 112 : le deductor et ses descendants "seraient automatiquement invités à servir de patroni à la colonie». E. Deniaux a attiré notre attention sur le fait que les Claudii étaient soucieux de se constituer des clientèles, et particulièrement Ti. Cl. Nero qui faisait quasiment du "démarchage": Verl.. Paterculus, II, 75, 1, pour des événements de l'année 41 en Campanie. 


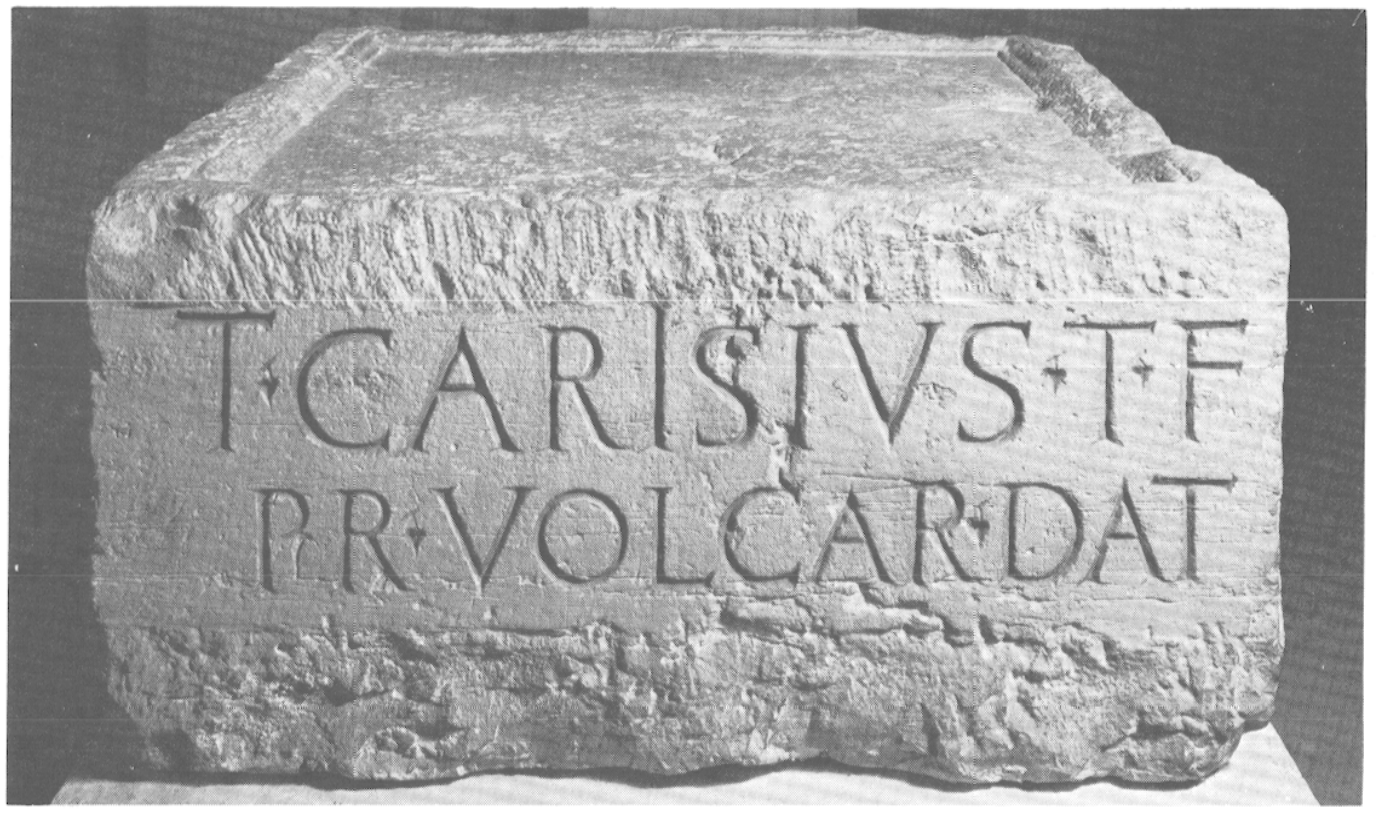

Fig. 3 - L'inscription d'Avignon.

L'une des questions que pose cette reconstitution, c'est celle des rapports entre l'«unité» volque arécomique et les "sous-unités» qui la composent. Elle n'a rien d'extraordinaire : la formation des confédérations celtiques rassemblant des peuples de moindres dimensions est bien connue; Strabon nous indique, par exemple, que l'armée salyenne était composée de dix unités juxtaposées (IV, 6,3) qui renvoient à dix "peuples» différents. Ceux-ci, tout en possédant leurs propres institutions, n'en reconnaissaient pas moins l'autorité des rois salyens. Peut-on, chez les Volques, trouver la trace de telles institutions? Voilà qui nous conduit à parler de T. Carisius.

\section{LE CAS DE T. CARISIUS, PRAETOR VOLCARUM}

Une pierre (fig. 3), découverte sur le rocher de Notre-Dame des Doms à Avignon, porte l'inscription: T.CARISIVS T.F.|PR.VOLCAR.DAT. ${ }^{21}$. C'est le socle d'une statue, dont l'encastrement a subsisté. Les critères paléographiques suggèrent une datation de la fin de la république ou de l'époque triumvirale, à la rigueur des débuts du règne d'Auguste.

21 R.A., I, 1844, p. 478-479, C.I.L., XII, 1028.
Cette inscription a fait l'objet de nombreux commentaires ${ }^{22}$. Si elle a retenu l'attention, c'est parce que le T. Carisius, fils de Titus, évoqué ici porte le gentilice de personnages connus à cette époque. Le premier attesté dans nos sources s'appelle T. Carisius. Il est magistrat monétaire de César en 46 avant J.-C. et n'est connu que par des monnaies ${ }^{23}$. Un second s'appelle simplement Carisius. Il est cité par Appien (B.C., V, 111, 463) qui le mentionne en compagnie de Titinius. On le considère comme un des légats de la flotte d'Octave en 36 avant J.-C., mais il fait partie des partisans d'Octave peu connus $^{24}$. Le troisième est le célèbre P. Carisius, légat d'Auguste en Espagne (Lusitanie) lors des guerres contre les Cantabres et les Astures, entre 27 et 25, puis fondateur de Mérida en 2525. S'il faut réduire le nombre de ces personnages, le deuxième

22 D'abord, en raison d'un développement défectueux : PR. VOLC(ano) AR(am) DAT proposé par C. CAVEdoni, Bull. Ist. di Corr. Arch., 1860, p. 208 et qui fit des adeptes comme Ilerzog et Jullian. IIirschfeld prit position en faveur de la bonne lecture dans le C.I.L., XII (p. 381).

23 Crawford, p. 475-476.

$24 \mathrm{R}$. Syмe, La révolution romaine (trad. fr.), Paris, 1967, p. 227 et p. 550 n. 47.

25 Dion Cassius, LiII, 25, 8; en dernier lieu, P. Le Roux, L'armée romaine et l'organisation des provinces ibériques d'Auguste à l'invasion de 409, Paris, 1982, p. 66-71. 
pourrait être identifié au troisième plutôt qu'au premier $^{26}$.

Certains ont voulu intégrer dans cette famille le préteur des Volques en l'identifiant au triumvir monètaire de César ${ }^{27}$, puis en faisant des Carisii, considérés dans leur ensemble, des sénateurs gaulois $^{28}$. Le dernier auteur qui, à notre connaissance, s'est penché sur la question, Y. Burnand, voit dans les Carisii des sénateurs de Narbonnaise; quant à leur lieu d'origine, après avoir balancé entre les Volques et les Helviens, donc avoir douté du rapprochement entre le magistrat monétaire de César et le préteur des Volques, il revient non seulement à l'origine nimoise mais aussi à l'assimilation possible des deux personnages ${ }^{29}$.

A supposer (car rien ne permet une certitude absolue) que l'inscription d'Avignon concerne un préteur des Volques Arécomiques, voilà qui conforterait les hypothèses que nous avons présentées : la personnalité juridique du peuple serait prouvée par la magistrature suprême exercée par T. Carisius. Retrouver ce dernier triumvir monétaire de César montrerait l'accession rapide d'un Volque aux honneurs, promotion supérieure à celle que l'on connaît par exemple pour des Allobroges ${ }^{30}$.

Sans exclure absolument cette hypothèse, n'occultons pas les difficultés qu'elle présente. Tout d'abord, quand et comment ces Carisii volques auraient-ils reçu la citoyenneté romaine? On ne peut envisager qu'une date haute puisque le père de "notre» préteur-triumvir monétaire est citoyen et se prénomme Titus. Dans ce cas, on s'étonne que luimême et ses descendants ne portent pas le gentilice d'une grande famille romaine comme les Valerii des

26 R. Syme, Roman Papers, p. 852 n. 1, et Rév. rom., ibid. Mais T.P. Wiseman, New Men in the Roman Senate, Oxford, 1971, p. 221, no 104, refuse cette identification.

27 E. HÜBNER, Exempla scripturae epigraphicae latinae, Berlin, 1885, p. 12, no 34 (fortasse); IIraschfeld, C.I.L., XII, p. 133; G. AlföldDY, Fasti Hispanienses, Wiesbaden, 1969, p. 131 .

28 Wiseman, New Men, p. 221, qui leur attribue Avignon comme origo; A. Chastagnol, Les sénateurs d'origine provinciale sous le règne d'Auguste, Mélanges Boyancé, Rome, 1974 , p. 163.

29 Les hésitations exprimées dans Sénateurs et chevaliers romains originaires de la cité de Nimes sous le HautEmpire, MEFR, 87, 1975, p. 695-697 ont été levées depuis lors : Epigrafia e ordine senatorio, II, p. 418, nos XXVIII et XXIX (avec l'assentiment de M. Le GlaY, p. 435). Y. Burnand a repris l'analyse dans sa thèse inédite Primores Galliarum, sénateurs et chevaliers romains originaires de Gaule de la fin de la république au $n I^{e} s$. : l'origo nimoise lui semble la meilleure hypothèse.

30 Ci-dessus, note 5.
Helviens ou les Pompeii des Voconces. A supposer qu'ils aient pris le nom d'un intermédiaire qui les aurait recommandés, encore faudrait-il connaitre des Carisii bien en vue dans la classe dirigeante romaine dans la première moitié du ier $s$. av. J.-C., ce qui n'est pas le cas.

Or, si l'on récapitule les noms des triumvirs monétaires de César entre 49 et 45 , on y trouve essentiellement des descendants de nobiles et des fils de sénateurs ${ }^{31}$; rares sont les représentants des milieux non sénatoriaux et il s'agit toujours d'Italiens comme L. Hostilius Saserna de Crémone ou M. Cordius Rufus de Tusculum. Le triumvir T. Carisius, de famille jusqu'alors non distinguée, a donc plus de chances d'appartenir à une gens italienne qu'à une famille transalpine.

Ce nom que R. Syme dit "rare et intéressant, d'origine non latine" (i.e. non originaire du Latium $)^{32}$ s'insère dans une liste de gentilices italiens de même formation et qui, à l'exception de Numisius et Calvisius un peu répandus, brillent par leur rareté : Apisius, Atisius, Larisius, Mimisius, Petisius $^{33}$. De ces dérivés en -isius, on ne trouve aucun exemple chez les Volques sinon un Apisius très douteux à Nimes et une Albisia Cn. f. Secunda ${ }^{34}$. On peut donc écarter l'hypothèse d'une "romanisation" de noms volques faite sur place lors de l'obtention de la civitas par les mécanismes de droit latin : Carus n'a pas donné Carisius; on attend plus KariusCarius, Kareius-Karius ou Carinius. Il faut donc se décider pour une origine italique. J. Suolahti, qui la préconisait, proposait Volci comme berceau ${ }^{35}$. Pour R. Syme, il s'agirait de Minturnes où plusieurs attestations révèlent un fort noyau de Carisii, vraisemblablement local, mais il existe aussi à Cumes un notable du nom Cn. Carisius L. f. ${ }^{36}$.

31 Nobiles : L. Valerius Acisculus (en 45), A. Licinius Nerva (47); fils de sénateurs : D. Iunius Brutus (49), C. Vibius Pansa (49), Man. Acilius (48), Ser. Sulpicius Rufus (48), L. Plautius Pancus (48), L. Papius Celsus (47), Lollius Palikanus (46), C. Antius Restio (46), C. Considius Paetus (45).

32 Rév. rom., p. 550 , n. 47.

33 Apisius : 17 occurrences; Atisius : 4; Larisius : 2; Mimisius : 14; Petisius : 13.

34 C.I.L., XII, 3415 et 3394.

35 J. Suolahti, The Junior Officers of the Roman army in the Republican periode, a study of a social structure, Ilelsinki, 1955, p. 286 et $350, n^{\circ} 47$.

36 R. Syme, More Narbonensian senators, Zeitschrift für Papyrologie und Epigraphik, 65, 1986, p. 5; J. Johnson, Excauations at Minlurnae, II. Inscriptions, Rome-Philadelphie, 1933, p. 55-56. Cn. Carisius L.f. est mentionné sur l'inscription en mosaique (Garrici, Mon. Ant., 22, 1913, col. 764) d'un pavement à "cocciopesto" de la fin de la république; le personnage est préteur. Cf. la thèse inédite de M. Cébeillac, 
La répartition des Carisii en Gaule méridionale vérifie apparemment cette présomption :

\begin{tabular}{|c|c|}
\hline CIL, XII, 317 & (一) Carisius M.f. T (?) (-)turio \\
\hline$C I L, \mathrm{XII}, 842$ & $\begin{array}{l}\text { Carisius Alexander } \\
\text { Carisius Amphion } \\
\text { Carisius Damas } \\
\text { Carisius Heracla }\end{array}$ \\
\hline CIL, XII, 416 & $\begin{array}{l}\text { M. Carisius Maximinus } \\
\text { M. Carisius Pacatus }\end{array}$ \\
\hline$C I L$, XII, 1556 & L. Carisius Serenus \\
\hline CIL, XII, 1772 & Carisia Bassiana \\
\hline CIL, XII, 1028 & T. Carisius T.f. \\
\hline$C I L, X I I, 2750$ & L. (?) Carisius (- - Maternus \\
\hline CIL, XII, 3957 & Sextia Sex. f. Carisia \\
\hline$I L G N, 450$ & Carisia L.f. Servata \\
\hline CIL, XIII, 8055 & T. Carisius T.f. Volt. \\
\hline CIL, XII, 4683 & L. Carisius L.f. Fronto \\
\hline$C I L$, XII, 4538 & L. Carisius L.l. Iucundus \\
\hline
\end{tabular}

Même en augmentant cette liste de quelques éléments peu sûrs ${ }^{37}$, on ne trouve ni à Nimes ni chez les Volques l'indication d'un rameau vivace autour des Carisii. Par ailleurs, les documents les plus anciens - outre le nôtre - concernent deux Narbonnais et un vétéran d'Alba, Titus fils de Titus (ce qui mérite l'attention).

Pour conclure, la probabilité est faible d'une origine volque des Carisii et d'une romanisation sur place. Egalement mince, la possibilité d'une obtention de la civitas virtutis causa à haute époque : on ne voit pas de grand personnage italien de ce nom susceptible de l'attribuer ou de la faire attribuer. Ne restent donc que deux solutions :

1. Un soldat volque reçut la citoyenneté virtutis causa durant les guerres césariennes ou peu après lorsque la gens Carisia connaissait un éclat qui, comme pour d'autres familles de l'époque, devait peu durer. Il eut (ou il avait) un fils qui devint praetor Volcarum. On ne saurait établir une chronologie assurée : un auxiliaire peut recevoir la civilas à

Les magistrats des cilés italiennes sous la république : le Latium et la Campanie, éd. dact., p. 682-684, qui rappelle également que l'épave A de la Jaumegarde à l'ouest de Porquerolles (Gallia, 27, 2, 1969, p. 479), chargée presque exclusivement d'amphores Dressel 1, a donné un opercule de pouzzolane marque du nom de L.CARISI, "lecture plus probable que le CARISIAN(us) proposé par l'auteur de la découverte». La proximité de Minturnes et de Cumes est un argument qui plaide en faveur d'une origine du sud du Latium ou de Campanie plutôt que de Volci.

37 ILGN 414 est selon nous très incertaine; il en va de même pour C.I.L., XII, 3415, inscription perdue dans laquelle nous restituerions Chrysius plutôt qu'Apisius ou qu'«à la rigueur" Carisius, comme le propose Ilirschfeld. région de la côte provençale

Arles

Marseille ( $\mathrm{II}^{\mathrm{e}} \mathrm{s}$.)

Vaison (sevir augustal)

Valence ( $\mathrm{II}^{\mathrm{e}}-\mathrm{IIII}^{\mathrm{e}} \mathrm{s}$.)

Avignon (praetor Volcarum)

Volques (partie orientale du territoire)

Volques (Nimes)

Volques (Nimes)

Alba (vétéran de la $I^{\text {re }}$ légion, début du I $^{\text {er }} \mathrm{s}$. après $\mathrm{J}$.-C.)

Narbonne (début du I ${ }^{\text {er }}$ s. après J.-C.)

Narbonne (début du I ${ }^{\text {er }}$ s. après J.-G.)

45 ans et avoir déjà un fils de 25 ; mais, sauf à tirer sur la corde, l'inscription a peu de chances d'être antérieure aux alentours de 30 .

2. Si on exclut le destin individuel (un Italien, T. Carisius, émigré motu proprio en Transalpine, devient ou voit son fils devenir préteur des Volques), rien n'interdit de penser que le père ou le fils ait pu faire partie de la déduction de Tiberius Nero et occuper ultérieurement (peut-être rapidement) cette charge.

En définitive, ce qui nous intéresse, c'est de constater que, selon toute vraisemblance (l'aventure personnelle d'un homme étant arbitrairement écartée - sinon à quoi bon écrire?), la charge de praetor Volcarum subsistait durant l'époque triumvirale, voire au début du principat d'Auguste.

\section{L'ÉPOQUE TRIUMVIRALE ET AUGUSTÉENNE}

$\mathrm{Si}$ on nous en croit, une colonie latine fut déduite à Nimes en 45 (ou 44). Elle frappa monnaie à légende NEM COL ${ }^{38}$ (fig. 4). Cette émission, cependant, ne semble pas avoir concurrencé le monnayage

38 L'émission comprend une obole en argent (tête casquée au droit, NEM COL dans une couronne au revers) et deux bronzes avec un seul droit (tête casquée) et deux revers majoritaires : palme et urne renversée (allusion évidente à la source), personnage debout à g. appuyé sur une colonne et tenant une patère au-dessus de deux serpents, Hygie-Valetudo ou encore SALVS, plutôt que "la colonie sacrifiant", ce que confirme une pièce trouvée à Nages en un exemplaire (M. Py, Acta Numismatica, IV, 1974, p. 128) : "R/ colonie assise, patère à la main ; serpents, siège", ce qui correspond aussi à des 

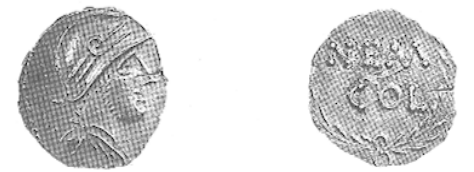

b

C
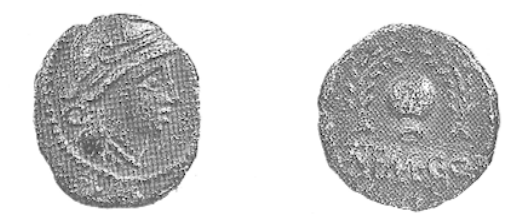

d
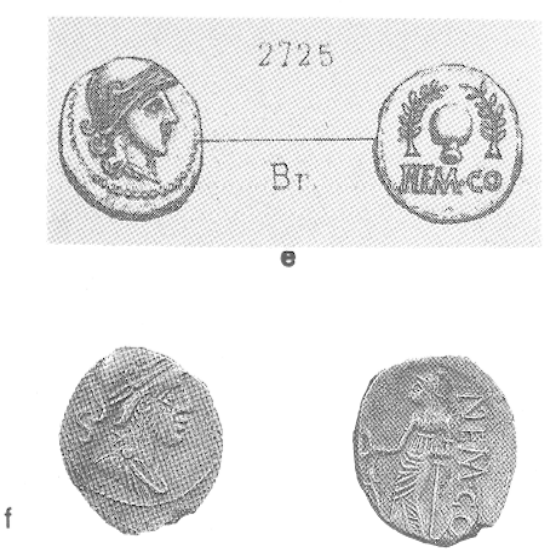

g

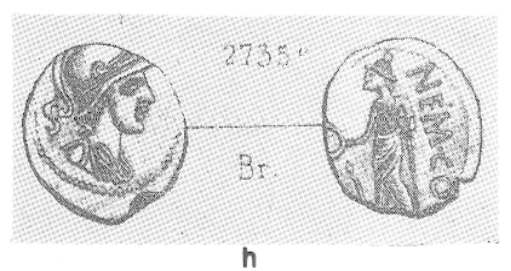

Fig. 4 - Le monnayage NEM COL : a, b, argent; c, d, e, premier petit bronze; f, g, h, second petit bronze.

représentations numismatiques de SAI.VS : par ex., R.I.C., I, 1984, p. 153, sans serpents; avec serpents : II. MarTINGLY, Coins of the Roman empire in the Br. Museum, III, p. 378 (et autres).

La datation de cette série n'est pas assurée. On la compare ordinairement à un monnayage de Cavaillon comprenant également une "obole" d'argent (et peut-être une diobole) et deux petits bronzes. Aucune similitude n'existe quant à l'iconographie : c'est le poids qui les rapprocherait (en dernier lieu, G. B. RogErs, Le système monètaire en Gaule de 43 à 23 avant J.-C., Revue Numismatique, 28, 1986, p. 83-93). La série cavaillonnaise présente l'avantage d'être datée, non par la légende LEPI qui se réfère à une titulature LEPIDA qui, si elle a chance de remonter aux années du gouvervement de Lépide en Transalpine (43-42), donne seulement un terminus post quem pour le monnayage, mais par la représentation sur l'obole et sur un petit bronze de la cornucopia qui semble liée au triumvirat avec des légendes connues par ailleurs portant le nom d'Antoine, de Lépide ou de César le Jeune et la mention III VIR RPG: Crawford, no 494, p. 13 à 15 . Dans les décennies suivantes, la corne d'abondance ne se retrouve jamais seule.

Or, si l'émission nimoise a d'abord été assignée par les
VOLC AR(EC) à l'extérieur de Nimes ${ }^{39}$, où, par ailleurs, est connue une autre frappe, représentée par fort peu d'exemplaires, qui attesterait l' "autonomie» d'une des autres communautés Volques ${ }^{40}$.

La déduction n'entraîna pas à Nimes les remous qu'on peut restituer à Vienne après la mort de César et qui ne sont pas sans parallèle ${ }^{\mathbf{4 1}}$. A en juger par le monnayage et par l'inscription de Carisius, l'identité de chacun des oppida latina ne fut pas mise en cause et la structure confédérale illustrée par le préteur des Volques continua d'exister.

archéologues à une date proche de 40 (D. Roman, La fondation ..., p. 100 et 103), plus récemment M. PY (étude inédite) la place aux environs de 30-20; J.-L. Fiches et J.Cl. Richard ("L'émission ...", p. 386) : «l'apparition des monnaies à légende VOLC et VOLCAE (se constate) dans le deuxième tiers du $\mathrm{I}^{\mathrm{er}} \mathrm{s}$. avant notre ère; celle des monnaies NEM COL à la mème époque est moins assurée et peut n'être intervenue que dans les années 30-20\%.

Tout cela est donc flou. Aux dires de J.-B. Giard luimême (qui avait suggéré le premier une possible correspondance pondérale avec l'émission de Cavaillon : Le monnavage..., p. 52, il faut étudier des lots nimois et cavaillonnais composant des échantillons statistiquement valables, ce qui n'a pas èté fait jusqu'à présent, y compris par Rogers. Pour nous, constatant que les datations proposées se fondent sur des donnees stratigraphiques relatives sans point d'ancrage en chronologie absolue (comment d'ailleurs le trouver à 20 années près?), connaissant aussi le décalage entre le matériel numismatique et le matériel céramique trouvés dans des couches archéologiques (le premier étant généralement plus ancien que le second), persuadés enfin qu'à l'heure actuelle l'habitude est de dater trop bas, nous inclinons en faveur d'une datation aux environs de 40-30.

En ce qui concerne Cavaillon, rien n'interdit de penser qu'à l'instar d'Antibes (Strakon, IV, 1, 9), qui porte aussi sur son monnayage la mention $\Lambda \mathrm{E} I \mathrm{I}$, elle a bénéficié d'une mesure prise en sa faveur par le triumvir. Mais ajouter Nimes à ces deux villes sur la foi de comparaisons numismatiques peu assurées, c'est une extrapolation.

39 J.-L. Fiches, J.-Cl. Richard, ibid., p. 384-385, avec nuances (données de l'Ermitage d'Alès).

40 Ibid., pour l'émission en argent à légende AMBR(ussum) au revers dans les cantons d'une roue. La plus forte relation s'établit avec les émissions NEM COL, particulièrement avec la division en argent (p. 383). Pas de données stratigraphiques.

41 E. Deniaux a analysé un cas semblable: Un exemple d'intervention politique : Cicéron et le dossier de Buthrote en 44 avant J.-C., Bulletin de l'Association G. Budé, 1975, p. 283-296. Les Buthrotiens chassèrent les colons et leur chef. Dira-t-on qu'à Nimes l'assimilation fut facilitée par la présence de citoyens romains déjà nombreux, si on en juge par la fréquence des gentilices Pompeius (cf. note 9) mais aussi Marius? De nombreux Arécomiques auraient bénéficié précédemment de la civitas virtutis causa. Surtout, il est possible que les terres assignées aux colons aient été celles reprises aux Massaliotes. 
C'est à ce point que se place le passage de Strabon IV, 1, 12. Rappelons la traduction qui nous paraît correcte :

"La métropole des $A$ récomiques est Nimes; bien inférieure à Narbonne pour la population étrangère et commerçante, elle l'emporte sur elle par son corps de citoyen. En effet, lui sont assujettis vingt-quatre oppida de même appartenance ethnique, à la population remarquable ${ }^{42}$, qui lui paient tribut mais qui jouissent également du droit latin, de sorte que ceux qui ont exercé l'édilité ou la questure à Nimes deviennent citoyens romains; et de ce fait, ce peuple échappe même à la juridiction des gouverneurs de Rome».

Au début du texte, Nimes est déclarée «métropole». Dans la langue de Strabon, le terme désigne le centre qui se trouve à la tête d'un peuple ou d'une confédération. Il n'est employé que pour Nimes, Vienne (IV, 1, 11) chez les Allobroges, Nemossos chez les Arvernes (IV, 2, 3) et Duricortora chez les Rèmes $(\mathrm{IV}, 3,5)$. Il se réfère toujours à un état préromain, comme on le voit au livre $\mathrm{V}$ pour Milan, métropole des Insubres (V, 1, 6), Corfinium pour les Péligniens (V, 3, 1) ou Pekelia pour les Lucaniens (VI, 1, 3). Lorsque le terme est employé au sens

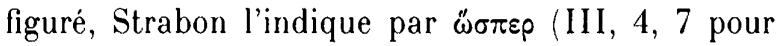

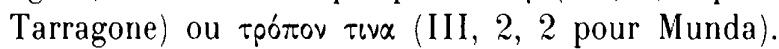
Tous les exemples montrent qu'il s'agit là d'une structure indigène, pas forcément lièe à une forte agglomération (Vienne et Milan étaient de simples villages $)^{43}$. Passons : le seul intérêt du mot est d'indiquer clairement quel centre était à la tête du peuple arécomique et qu'il s'agit de la persistance d'une structure autochtone.

Strabon télescope les événements, comme il lui arrive fréquemment. Il passe d'un état ancien à l'état présent, faute de disposer des sources qui lui fourniraient les étapes ${ }^{44}$. Des vingt-cinq oppida latina césariens jouissant, au sein de la confédération volque arécomique, d'une personnalité juridique, on

42 Selon P. Thol.t.ARD, La notion de civilisation dans les livres III et IV de Strabon, thèse sous presse dans les Annales littéraires de l'Université de Besançon, il convient de distinguer

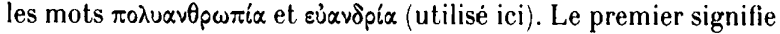
l'importance numérique d'une population (par ex., des Belges : IV, 4,3 ). Le second se référerait à l'importance du nombre des citoyens romains; il est employé pour Tarragone, Lyon, Cadix, Rhegion.

43 P. Tholland, ibid.

44 Rappelons qu'il ignore totalement le phénomène de colonisation en Transalpine, mème en ce qui concerne Narbonne, dont il tait aussi, dans la notice qui la concerne, la qualité de chef-lieu de la province. arrive à une situation que Pline traduit par le mot adtribuere : XXIIII (oppida) Nemausensibus altribu$t a^{45}$.

Le changement, c'est d'abord une modification institutionnelle. Le passage doit s'interpréter $a$ contrario. Jusqu'alors, les vingt-quatre oppida arécomiques (outre Nimes), jouissant du droit latin, bénéficiaient de sa disposition bien connue accordant la citoyenneté à quiconque exerçait au sein de la communauté une magistrature inférieure (édilité ou questure). C'est cette disposition qui fut supprimée : il fallut désormais exercer la magistrature non pas chez soi mais à Vimes. Voilà qui explique le terme de Pline : oppida ignobilia. Il ne s'agit pas d'établissements inconnus ou peu renommés mais d'établissements "déclassés", ayant perdu la personnalité juridique. Leurs citoyens conservent le bénéfice du droit latin mais ils doivent gagner la civitas à Nimes. Cette mésaventure frappa les vingt-quatre centres arécomiques et dix-neuf autres répartis dans la provincia.

Nous sommes tentés d'aller plus loin et de reconstituer un système institutionnel à deux étages. Avant le déclassement dont nous venons de parler et depuis l'octroi du jus Latii, il y avait, d'une part, les magistratures exercées dans les vingt-cinq oppida latina, sur la lancée des institutions de type indigène; d'autre part, une ou des magistratures exercées au niveau confédéral (sans doute à Nimes). Peu

45 Pline fournit un décompte précis : $29+14$. Cela suppose que sa source, la formula, le permettait. Plutôt que d'imaginer qu'elle ait comporté une rubrique spécifique (comme celle énumérant les oppida stipendiaria de Bétique et de Tarraconnaise : IIN, III, 7 et 18), il est préférable de penser que, sur la liste alphabétique des (environ) 75 oppida latina, 43 avaient été rayés et (ou) suivis de la mention : Nem. attributum pour 24 d'entre eux, et $x$... attributum pour 19 autres. Si la formula a été refondue en 27 , il est probable que cette mesure de déclassement a été prise à une date ultérieure (cf. infra). Cette reconstitution suggère deux commentaires. D'abord, cela signifie $a$ contrario que les oppida non déclassés, y compris ceux qui ne furent pas chefs-lieux de civitates, ont conservé les moyens institutionnels de faire obtenir à leurs citoyens la civitas per magistratum sur place (ce qui expliquerait entre autres, le forum de Glanum et la dénomination respublica du $C I L, \mathrm{XII}, 1005$ ?). On relira, en ce sens, certaines réflexions de M. IIumbert, Le droit impérial : cités latine ou citoyenneté latine?, Klèma, 6, 1981, p. 207-226. D'autre part, si on ne peut se prononcer sur les 19 autres oppida déchus, la formule de Pline sicut XXIIII Nemausensibus attributa laisse supposer des attributions à telle ou telle cité, mais rien d'aussi important que ce qui a concerné Nimes (du moins numériquement); elle ne permet pas d'affirmer que les mesures d'attribulio furent décidées au mème moment pour les 43 oppida devenus ignobilia; le cas de Nimes aurait-il servi de référence ou de modèle (sicul ...)? 
importe le nom des magistratures des oppida lati$n a^{46}$. Des magistratures confédérales, nous avons un témoin : le praetor Volcarum. Comme dans d'autres cités (dans leurs chefs-lieux), elle évolua vers la collégialité et un habillage plus romain : à Nimes (CIL, XII, 3215), ce fut un praetor IIIIvir, puis le terme praetor disparut. Cependant, une des originalités nimoises consiste en la charge de praefectus vigilum et armorum, sous laquelle on ne sait trop quelle responsabilité exacte placer. Elle évoque, chez les Voconces, peuple fédéré dont la magistrature suprême demeura, jusqu'au III ${ }^{\mathrm{e}}$., celle du praetor, la préfecture praesidiorum et privatorum ${ }^{47}$. En outre, on a toujours remarqué, chez les Arécomiques, une nette séparation entre magistratures inférieures et supérieures : à une exception près, les inscriptions distinguent édiles et questeurs et, d'autre part, préfets des armes, pontifes et quattuorvirs ${ }^{48}$. On peut se demander si nous n'avons pas là le reflet d'institutions anciennes: au niveau du peuple, le pouvoir confédéral consistait en un binôme civil et militaire, traduit ensuite le premier par le terme de praetor, le second par une périphrase manifestant l'évolution des réalités et se référant au simple maintien de l'ordre. Mais le point essentiel demeure la prééminence du praetor.

On voit la "révolution» que décrivent ou qu'esquissent Strabon et Pline: le passage d'un système confédéral à un système centralisé au profit de Nimes; c'est elle qui rassemble désormais toutes les institutions et, même si en théorie la civitas per magistratum reste ouverte à tous les Arécomiques, la possibilité de l'obtenir se restreint singulièrement, ne serait-ce que par l'arithmétique. Mais il y a plus : lorsque Strabon écrit que les vingt-quatre oppida sont tributaires de Nimes, il faut prendre l'expression au pied de la lettre, c'est-à-dire considérer que

46 Peut-on ranger parmi elles l'édilité collégiale exercée à Murviel-lès-Montpellier par Sex. Vetto et C. Pcdo (CIL, XII, 4190: aetatis primae imperatoriae vel liberae reipublicae exeuntis)?

47 CIL, XII, 1368. Chr. Gounineau, Les fouilles..., p. $288-295$.

48 Y. Burnand, Sénateurs ..., p. 739-740. Une inscription récemment découverte sur l'oppidum de Saint-Vincent de Gaujac révèle le cursus complet d'un notable nimois du $\mathrm{I}^{\mathrm{er}} \mathbf{s}$. ap. J.-C. (plutôt de la première moitié) : après la publication des premiers fragments par J. Charmasson, R.A.N., 18, 1985, p. 389-392, cf. In. et M. Christol, Une importante découverte épigraphique romaine à l'oppidum Saint-Vincent de Gaujac, Rhodania, 21, 1987, p. 12-23: [Ap]oll/ini] / /.JAn/t]onius L(uci) f(ilius) Vo[l(tinia)] / Pater[nu]s, aedil[is] / prae[f(ectus)] [f]abrum, IIIIvir ad aerar(ium). C'est la première fois qu'est signalée une continuité entre l'édilité et les charges supérieures: l'exception qui confirme la règle?
Rome lui a accordé un privilège financier considérable, celui de collecter les impôts à son seul profit. Toutes ces dispositions la placent dans la catégorie des peuples les plus favorisés, celle des civitates foederatae. Et c'est exactement ce que précise Strabon: le peuple des Arécomiques échappe à la juridiction des gouverneurs. Il utilise la même expression pour les Voconces dont Pline atteste le statut ${ }^{49}$.

Le texte de Strabon et la rapide mention de Pline s'accordent sur un point : ils signalent à Nimes un phénomène exceptionnel, l'attribution de vingtquatre oppida. Sur les soixante-quinze oppida latina (environ) créés par César, quarante-trois furent déclassés et devinrent ignobilia, perdant leur personnalité juridique autonome. Parmi eux, dix-neuf, répartis (probablement) en différents points de la provincia, furent rattachés à telle ou telle cité. Ce qui a frappé les deux auteurs, c'est que tout un bloc fut attribué à une seule cité, Nimes. Autrement dit, l'opération fut double : une réduction drastique du nombre des oppida autonomes (près de $60 \%$ !) - mesure générale - et - mesure particulière l'attribution à Nimes de vingt-quatre de ces quarante-trois oppida. Du coup, Nimes retrouvait une juridiction très étendue, peut-être proche de celle des Arécomiques. D'après le terminus ante qu'on octroie au livre IV de Strabon, on peut placer ces décisions à une époque antérieure aux environs de 20 après J.-C. ${ }^{50}$.

La prudence nous invite à nous en tenir là et à donner la conclusion suivante : si notre analyse des institutions est juste, l'interprétation des textes conduit à placer l'attributio de vingt-quatre oppida latina à Nimes entre, grosso modo, 30 avant J.-C. et les débuts du règne de Tibère, c'est-à-dire durant le principat d'Auguste à quelques années près dans

49 Strabon, IV, 6, 4: "Les Allobroges et les Ligures sont soumis aux gouverneurs dépêchés en Narbonnaise, tandis que les Voconces, à l'instar des Volques autour de Nimes dont nous avons parlé, s'administrent eux-mêmes». PLINE, III, 37 : Vocontiorum civitatis foederalae.

50 Ce statut fédéré dura-t-il longtemps? On pourrait en douter du seul fait que Pline ne l'évoque pas, alors qu'il en fait mention pour les Voconces. Cependant, si on admet (cf. note 45 et infra) que cette disposition est un ajout à la formula de 27, on pourrait supposer que Pline a été frappé par l'attribution plus que par ce statut nouveau; en revanche, la formula de 27 entérinait la lex césarienne qui avait déjà accordé ou confirmé aux Voconces le statut fédéré. Peu importe d'ailleurs : la notion de "fédération" perdit rapidement son sens originel: A. N. Sherwin-White, The Roman Citizenship, Oxford, réédition 1973, p. 186-187, H. IIoRN, Foederati, Francfort, 1930, p. 42-59, etc. 
chaque sens. Nous pouvons nous sentir satisfaits : les aspects financiers, fiscaux et juridiques de l'opération rendent compte de la parure monumentale, des investissements précoces et exceptionnels que la cité a réalisés et de ses particularités épigraphiques.

\section{HYPOTHÈSES}

Tentons quand même d'aller plus loin. On l'a dit précédemment, le texte de Pline reflète la «stratigraphie" de la formula provinciae: à partir de textes pompéien et césarien, une refonte augustéenne qui ne subit ensuite que des modifications de détail prenant acte de réaménagements limités.

Si la refonte correspond au séjour d'Auguste en Gaule en 27 avanl J.-C. et si l'évolution qui avait conduit Nimes, oppidum latinum comme bien d'autres, à la Colonia Augusta Nemausensium avec domination sur un vaste territoire et attribution de vingt-quatre oppida jusqu'alors autonomes, si, donc, cette évolution avait été menée à son terme, Augusta Nemausensium n'aurait-elle pas été rangée sous la lettre A (à côté d'Augusta Tricastinorum - ̀̀ supposer que celle-ci soit de date haute) et non pas sous la lettre $\mathrm{N}$ ? Le rang alphabétique de Nemausus Arecomicorum suggérerait plutôt qu'au moment de la refonte de la formula, la cité, quoique colonie de droit latin, n'avait pas été dotée de la préeminence et des privilèges qu'elle obtint par la suite.

Cette prééminence et ces privilèges, nous sommes tentés de les attribuer au séjour du prince de 1613 avant J.-C. (Dion, LIV, 25, 1), durant lequel il prit des mesures de réorganisation en Espagne, dans les Gaules et les Germanies avant de regagner Rome en chargeant Drusus de l'administration des Gaules et du recensement des provinces. Ce serait à cette date que, mettant en œuvre une politique destinée à développer les villes, à y attirer les élites (et peutêtre aussi à réduire les facilités d'accès à la civilas), il aurait opéré cette double procédure de "déclassement" et d'attribution, dont la formula prit acte en rayant des noms d'oppida latina, ce qui permit à Pline de gloser sur leur déclassement et de les dénombrer. Pour autant, la formula ne revenait pas sur Nimes même : la titulature Augusta n'impliquait par un changement de droit.

Ce scénario explique-t-il tout? Certainement pas. S'il rend compte des textes, voire de l'épigraphie institutionnelle, il ne propose pas de justification historique. Nous rencontrons ici un problème de mélhode. A reprendre l'ensemble du dossier, nous avons été frappés par la rapidité avec laquelle ont été abandonnées des thèses forgées par un siècle et demi de réflexion. Les savants du XIX ${ }^{e}$, partant de la titulature Colonia Augusta, avaient supposé une déduction augustéenne, ce qu'ils étayaient à l'aide d'autres arguments : l'inscription de la porte, la Maison Carrée, les noms gréco-orientaux. Et surtout par le monnayage au crocodile. Or, sur la seule foi des émissions NEM COL et de leur datation hypothétique aux alentours de 43 , on admet communément que ce nom AVGVSTA ne représente qu'une faveur d'Auguste à l'égard d'une colonie fondée (ou élevée à ce rang) par son père ${ }^{51}$. Il faut dire clairement que rien n'est moins sûr.

Partons du monnayage au crocodile, qui «inonda" tout l'Occident et en dépassa les limites (fig. 5). S'il s'agit de commémorer le rattachement de l'Égypte, pourquoi la frappe porte-t-elle COL NEM? L'autre émission connue qui représente un crocodile (d'ailleurs sans entrave et seul dans le champ) et qui porte la légende AEGVPTO CAPTA ne mentionne ni ville ni colonie ${ }^{52}$, et c'est pourquoi tous les numismates et la plupart des historiens ont considéré qu'un lien puissant existait entre la Colonia Nemausus (ou Nemausensium) et la conquête de l'Égypte.

Si le crocodile symbolise l'Égypte, à quoi correspond la palme à laquelle il se trouve enchaîné ? A l'Apollon d'Actium ${ }^{53}$ ? Pas impossible mais compliqué. Rappelle-t-elle les présages concernant le jeune Octave que nous connaissons par des textes ${ }^{54}$ ? Ce serait bien allusif. Or, la palme est présente dans tous les monnayages à légende latine de Nimes et des Arécomiques. Elle figure devant le personnage en toge VOLC AREC et peut-être sous l'aigle de la

51 "Fables orientalisantes», écrit D. Roman, Dossiers IIist. et Archéologie, 55, 1981, p. 91-92.

52 Cf. la nouvelle édition du R.I.C., 1, 1984, p. 85-86: Sutherland ne donne aucune origine à ces monnaies ("Pergamon has been proposed, rather improbably $(. .$.$) ; it seems best$ to leave open the question "). En outre, ces pièces sont très rares.

53 D. Roman, Apollon... (cf. note 2).

54 Dion, XLIII, 41 (45 avant J.-C.) : lors de la bataille de Munda « un palmier, qui était sur le champ de bataille, avait poussé un rameau aussitôt après la victoire. Je ne prétends pas dire que ce ne fut pas là un présage, mais il n'était pas pour lui (César) mais pour Octave, petit-fils de sa sœur". Suétons, Aug., 94 : "Ce prodige fut, dit-on, le motif qui détermina le plus César à ne vouloir d'autre successeur que le petit-fils de sa sœur». Sú́tone, Aug., 92 : "(Auguste) transporta dans le sanctuaire de ses dieux pénates un palmier qui était né devant sa maison entre les joints de pierres et mit tous ses soins à le faire pousser». Un palmier poussa sur l'autel d'Auguste à Tarragone: Quintil.tifn, I. Or., VI, 3, 77. 


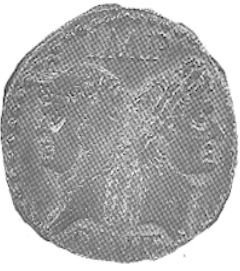

a.

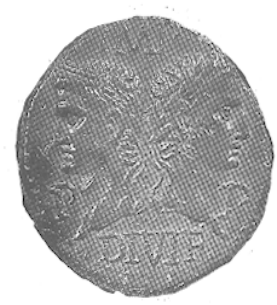

d

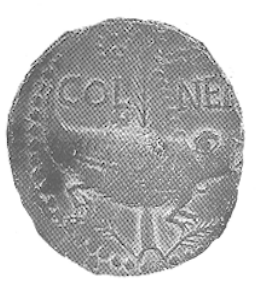

b

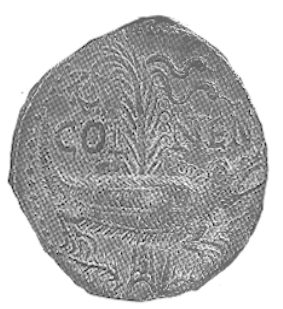

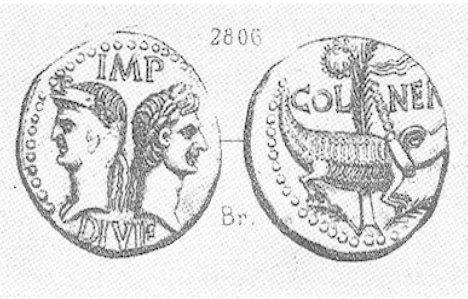

c

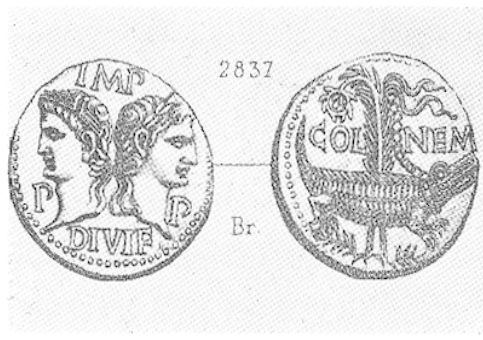

f

Fig. 5 - Monnayage au crocodile : a, b, c, première émission ;

$\mathrm{d}, \mathrm{e}, \mathrm{f}$, seconde émission.

même série (serait-ce l'explication de l'adoption de ce revers de Pomponius Rufus?), elle accompagne l'urne renversée de la série NEM COL; on la retrouve enfin sur les as. Le hasard paraît exclu mais comment rendre compte de cette omniprésence? Est-elle liée à la source que manifestent les autres représentations proches de VALETVDO ou de SALVS? Si les palmiers poussaient naturellement à Nimes, l'hypothèse d'un bois sacré s'imposerait mais, à en juger par les efforts désespérés de l'actuelle municipalité pour entretenir deux de ces arbres et faute de pouvoir prouver qu'il en allait autrement dans l'antiquité, mieux vaut abandonner à regret cette piste. La palme, le palmier se réfèrentils à Apollon? Le monnayage le plus ancien, à légende grecque NAMA $\Sigma \mathrm{AT}$, comportait un droit identique (ou presque) à l'Apollon du petit bronze massaliote au taureau, mais l'indice est fragile : il faudrait supposer qu'on est passé de la représentation explicite d'Apollon à une représentation symbolique, la palme. Par ailleurs, le culte d'Apollon n'a pas laissé à Nimes de traces notables, et rien ne suggère qu'on ait pu l'identifier à Nemausus, bien au contraire.

Donc, d'une part, la palme symbolise Nimes - sans que nous sachions pourquoi ${ }^{55}$; de l'autre, le

55 Dans le champ des hypothèses invérifiables, on ne peut exclure qu'Auguste, pour lequel la palme et le palmier avaient une signification (note 54), ait été sensible à cette relation entre Nimes et ce symbole - ou cet arbre. Roman plus que démonstration... crocodile lui est enchaîné; enfin, la légende NEM COL propage la gloire des colons nimois sous le double patronage de César le Jeune et d'Agrippa. Entre la COL NEM et la conquête de l'Égypte, il y a une relation forte.

S'agirait-il d'une participation à ces campagnes des colons de Tiberius Nero? C'est évidemment improbable, à une quinzaine d'années de distance. On se retrouve donc devant les questions que se posait, en 1883, Otto Hirschfeld : "Quelles raisons peuvent avoir conduit une ville comme Nimes à choisir un monnayage qui se réfère à la conquête de l'Égypte? Friedländer, suivi par Froehner, suppose que des vétérans des campagnes d'Egypte ont pu être installés dans cette colonie. Robert a avancé de bons arguments pour réfuter cette théorie; il rappelle les témoignages incontestables de Strabon et de Pline selon lesquels, à l'époque d'Auguste, Nimes n'était pas colonie romaine mais latine, alors qu'une deductio veleranorum normale comportait automatiquement l'octroi de la citoyenneté. Cependant, selon moi, il ne s'agissait pas de vétérans romains, plutôt de grecs égyptiens, particulièrement de soldats qui avaient servi dans l'armée et dans la flotte d'Antoine dont l'éloignement d'Égypte pouvait être souhaitable ${ }^{56}$.

56 Die Crocodilmünzen von Nemausus, Wiener Sludien, 5,1883 , p. $319-322$. 
La thèse de Hirschfeld n'a en soi rien d'impossible à condition qu'on lui apporte un aménagement : il ne s'agit pas d'une première déduction mais d'une seconde, semblable à celle que César réalisa à Narbonne en 45-44 après la fondation originelle de 118. Or, on sait qu'Octave-Auguste envoya des colons dans d'anciennes colonies. Deux "campagnes" de colonisation sont attestées par les Res Gestae, 16 et par Dion Cassius LI, 4 et LIV, 23. Ces textes s'accordent pour situer la première juste après la bataille d'Actium et la seconde (les deux textes divergent légèrement) entre 16 et 14. Dans le premier cas, on ignore la localisation précise des déductions; pour le second, Dion écrit qu'Auguste "colonisa plusieurs villes en Gaule et en Espagne", Or, pour la Gaule, on ne saurait proposer un seul nom, sauf précisément à admettre une nouvelle vague d'arrivants dans telle ou telle cité déjà colonisée ${ }^{57}$. Pourquoi pas dans la Nimes, colonie césarienne, déduite par Tibérius Nero ? Voilà en tout cas qui expliquerait le patronage de C. Caesar, fils du deductor ${ }^{58}$.

Ces interprétations historiques et numismatiques trouvent-elles des appuis extrinsèques? Dans l'étude topographique déjà mentionnée, J. Benoit indique que la "ville verte» pré-augustéenne (celle que nous avons rattachée à la déduction césarienne) reçut peu après une importante extension (la "ville violette»), elle aussi rigoureusement organisée et qui, en quelque sorte, "cerne" l'urbanisme public augustéen (fig. 2). Une seconde déduction la justifierait.

Peut-on trouver dans le monnayage nimois au crocodile un élément chronologique? On dira d'emblée que, plutôt que de commémorer de façon immédiate l'événement, il peut aussi bien le commémorer d'une façon large et rétrospective. En d'autres termes, l'apparition de ces monnaies fournit le terminus ante quem de cette déduction supposée. Or,

57 C'est aussi le cas de l'Italie : L. Keppie, Colonisation ..., p. 17 : "There must be some suspicion that the title Augusta reflects additional veteran settlement between $27 \mathrm{BC}$ and $14 \mathrm{AD} »$; cf. aussi p. 83. Augst s'intitule Augusia Rauricorum et fut cependant fondée par L. Munatius Plancus en 43.

58 On ne saurait éliminer l'hypothèse selon laquelle une telle opération se serait déroulée en 20-19 lorsque Agrippa séjourna en Gaule après avoir passé deux ans en Orient, pendant lesquels il avait rencontré de difficiles problèmes de colonisation. Cependant, CIL, XII, 3153 et 3154 n'attestent en rien un patronage d'Agrippa sur .Nimes. Sur un aussi long décalage entre Aclium et la colonisation, plusieurs présomptions, par ex. pour Patras et Beyrouth, fondées en 15 avant J.-C. d'après la Chronique d'Eusèbe: bibliographie dans M. Rondaz, Marcus Agrippa, BEFAR, 1984, p. 431-433. Cf. aussi E. Dfniaux, Un exemple... la date des premières frappes fait l'objet de controverses entre les partisans d'une chronologie haute (28-27) et ceux d'une chronologie basse (pas plus haut que 20 , plutôt après 18$)^{59}$.

Rappelons les arguments récemment invoqués pour appuyer une date haute. En premier, la titulature IMP DIVI $F$ impliquerait que la frappe de la première émission serait antérieure au 16 janvier $27^{60}$. Mais si l'on suppose que la légende reflète fidèlement le statut politique du prince, pourquoi les émissions ultérieures ne comportent-elles que l'adjonction PP sans que jamais n'apparaisse le surnom Augustus? On a aussi expliqué l'association d'Auguste et d'Agrippa sur le droit par le fait qu'une telle image symbolisait l'égalité des pouvoirs des consuls de 28 avant $\mathbf{J}$.-C., dans une ambiance de "restauration républicaine $\|^{81}$. En fait, l'installation du prince sur le droit des monnaies s'était faite à la fin de l'époque césarienne et durant la guerre civile : on doit l'interpréter comme une manifestation des imperatores pour marquer leur importance sinon leur "prépotence», exaltation de l'individu et sa position $^{62}$. L'interprétation "républicaine» a donc quelque chose de paradoxal.

Lorsque, en 38-37, apparurent deux effigies, c'étaient celles de César divinisé et de César le $J^{J} u^{63}$. Héritage familial et héritage politique justifient cette association, explicite dans la dénomination du prince : IMP. CAESAR DIVI F. Dans le contexte des images politique, le droit des monnaies de Nimes symbolise donc - si l'on ose dire - l'«égalité monarchique" entre Auguste et Agrippa, ce dernier appelé aux côtés du prince, ce qui se conçoit difficilement en dehors d'une situation

59 Immense bibliographie. En dernier lieu, pour la date haute, Roddaz, notamment p. 595-597; pour la chronologie basse, $R I C, 1$, nouvelle édition, p. 51 (avec renvois bibliographiques), pour lequel la première émission doit être datée entre 20 et 10 environ. Cf. aussi C. H. V. Sutherland, C. M. KraAy, Cal. of coins Rom. Emp. in the Br. Mus., Oxford, 1985, pl. 10.

60 Par exemple, H. Zennacker, La trouvaille de Villeneuve-au-Chatelot (Aube), Trésors Monélaires, VI, 1978, p. 56 .

61 Roddaz, p. 207 (et n. 43) s'appuie sur le faux parallèle d'une monnaie de Sinope qui remonte à 27 (et non à 28) : en fait, le droit est attribué à Auguste et le revers à Agrippa. Sur les monnaies de $38-37$ examinées p. 594, le principe est le même.

62 F. Millar, State and Subject. The impact of monarchy, Caesar Auguslus, Seven Aspects, F. Millar and E. Segal ed., Oxford, 1984, p. 44-45.

63 On les retrouve vers $28-27$ (?) sur le monnayage de Lyon: Sutherland-Kraay, pl. 26 ; J.-B. Giard, Le monnayage de l'alelier de Lyon des origines au règne de Caligula, p. 17 et nos 5-7, pl. IV-VII. 
institutionnelle précise (plus même que familiale). Dans le dossier réuni par J.-M. Roddaz, les rapprochements les plus forts semblent correspondre à la "co-régence", mais il faut observer que, si tous deux apparaissent côte à côte sur le revers, Auguste garde la prééminence sur le droitt. Ce n'est qu'à partir de 19-12 avant J.-C. qu'ils furent associés sur le droit, et en province ${ }^{65}$. Dira-t-on que les émissions de Nimes sont un monnayage local de chronologie plus haute? Difficile, vu leur expansion. Voilà donc qui ajouterait force à la conviction exprimée par A. Chastagnol ${ }^{66}$ : "pour que les effigies d'Auguste et d'Agrippa soient disposées côte à côte sur un plan d'égalité mais avec la titulature propre au seul Auguste, il faut qu'Agrippa ait été associé officiellement aux pouvoirs de son ami, notamment à la puissance tribunicienne, ce qui advint seulement en 18 avant J.-C., alors qu'il était devenu depuis trois ans le gendre du prince par son mariage avec la jeune Julie" ${ }^{67}$.

64 Ce que note Roddaz lui-même (p. 603). On peut prendre la mesure du phénomène en parcourant l'ouvrage de Sutherland et Kraay (note 59). On comparera avec le monnayage pour $($. . et $L$. César : ces princes apparaissent au revers entre 8 et 1 (ibid., pl. 9, $\mathrm{n}^{\circ}$ 156-160) avant d'occuper le droit après 2 après J.-C. à Tarraco (pl. 24, nos 1100-1101). Puis Tibère et Auguste sont associés dans le monnayage royal de Thrace entre 4 et 12 (?) : ibid., pl. 21, no 850 .

65 E.S. C. Robinson, Cal. Greek coins Br. Mus., réédition Bologne, 1965, p. 119, nos 36-39; M. Grant, From Imperium to Auctoritas, Cambridge, 1946, p. 137 sq. ; SutherLandKraAy, pl. 20, n" 896 (après 24 avant J.-C.); Roddaz, ibid., p. 606-607. C'est dans ce contexte provincial qu'on doit placer les monnaies d'Alabanda en Carie (citées par Roddaz, p. 606 ; B. V. Inean, Cat. Greek coins Br. Mus. : Caria and Islands, Londres, 1897, p. 5, $\mathrm{n}^{\circ}$ 28) : Auguste et Livie occupent le droit, Agrippa, Caius et Lucius sont figurés sur le revers. Même organisation à Magnésie (Sutherland-KraAy, pl. 32, n' 1358)

66 A. Chastagnol, Monnaies de Nimes au type du crocodile trouvées à Rennes, Annales de Brelagne, 73, 1966, p. 195. Voir les observations de J.-B. GIARD, Le trésor de Port-Haliguen, contribution à l'étude du monnayage d'Auguste, R.N., $6^{\mathrm{e}}$ série, 9, 1967, p. 124-126: tout en conservant la datation traditionnelle, l'auteur examine cette hypothèse avec beaucoup d'intérêt.

67 Rappelons aussi que, vérifiant une assertion de Friedländer, Ilirschfeld (cf. note 56) a observé que deux exemplaires des as de la première émission comportaient, dans la couronne suspendue au palmier, une marque $\lfloor\Delta$, soit une datation selon le calendrier d'Alexandrie, c'est-à-dire la quatorzième année à compter du 29 août 31 , donc 17-16. Il notait que la dédicace de la porte d'Auguste, datée par la huitième puissance tribunicienne, correspond à l'année qui commence le 26 juin 16 . Une correspondance s'établirait sur juillet et août 16 , mois qui coïncident avec le début du long séjour d'Auguste en Gaule et en Espagne. Tibère (selon nous patron de Nimes) l'accompagnait. Deux monnaies ne font pas le printemps et il peut s'agir d'une frappe spéciale. N'empêche.
Enfin, l'abondance des frappes n'est pas indifférente $^{68}$. Elle suppose une cité financièrement solide et peut-être dotée de l'indépendance par rapport au gouverneur provincial. N'oublions pas la faiblesse quantitative des monnayages locaux antérieurs ainsi que celle de toutes les frappes issues des autres villes de la province.

Le monnayage de Nimes s'inscrirait donc dans une série de mesures prises vers 16 : un nouveau statut, l'attributio des oppida, la titulature Augusta. Il s'agissait de constituer une cité puissante, dominée par une grande ville, dotée des moyens de se développer et à laquelle l'empereur offrit de surcroît ses murs et ses portes ${ }^{69}$.

Si l'on retient l'hypothèse d'une seconde déduction, celle-ci serait donc antérieure à 16-13. De combien d'années? Bien que les problèmes d'installation sur les terres coloniales aient pu exiger de longs délais, on ne saurait trop s'éloigner de l'annexion de l'Égypte, mais sans pouvoir proposer de chronologie précise.

Il existe cependant une présomption : la précocité du culte impérial auprès de la source de Nemausus, attesté par l'épigraphie dès 25 avant J.-C. ${ }^{70}$. Cette date paraît si extraordinaire en Occident qu'on cherche des explications détournées. Mais

68 La liste établie par M. Grant, From Imperium..., p. 71, demande à être reprise. Il n'est guère de fouille récente des débuts de l'Empire qui ne fournisse un contingent d'as de Nimes. La même obșervation a été faite pour le monnayage de Lyon: Giard, Lyon, p. 17.

69 On pourrait être tenté de placer ces mesures dans le fil des déclarations de l'empereur, Res Gestae, 16: "pour l'acquisition des terres que j'ai assignées aux soldats dans mon quatrième consulat ( 30 avant $\mathbf{J}$.-C.) puis sous les consulats de M. Crassus et de Cn. Lentulus Augur (14 avant J.-C.), j'ai payé des indemnités aux municipes». Mais ces faveurs excèdent une indemnisation. L. KePpIr, Colonisation, p. $115 \mathrm{sq}$., a signalé la rareté de tels dons. L'indépendance accordée à Nimes par rapport au gouverneur de la province fait échapper la civitas au contrôle du Sénat auquel la provincia avait ćtc் «renduc»en 22 (Dion, LIII, 12) et instaure donc la possibilité de liens directs avec l'empereur.

70 CII, XII, 3148 et 3149 : Auguste est consul pour la neuvième fois et désigné pour la dixième. Les inscriptions, à elles seules, n'indiquent pas un culte, mais les pierres qui les portent "étaient taillées pour s'adapter aux exèdres qui animent la paroi sud" du bassin de la source et semblent "appartenir à la phase initiale de la construction": P. Gros, L'augusteum ..., p. 129. Faut-il rappeler que, par ailleurs, Nimes détient le record en Gaule pour le nombre des inscriptions de seviri augustales, et surtout pour les Lares Augusti dont M. Hano (A l'origine du culte impérial: les autels des Lares Augusti, Aufstieg und Niedergang der Römischen Welt, II, 16.1, 1986, p. 2333-2381) vient de reprendre l'étude pour Rome, en montrant le caractère à la fois précoce et populaire. 
si nous acceptons l'hypothèse d'une déduction de contingents gréco-orientaux, une telle précocité se comprend. Elle s'accorde de plus avec l'interprétation récemment proposée par $\mathrm{P}$. Gros, voyant dans le jardin de la Fontaine un augusteum constitué sur le modèle des "sebasteia" orientaux et particulièrement sur celui que décrit, pour sa propre ville, Philon d'Alexandrie ${ }^{71}$. Même si l'augusteum s'est "monumentalisé» un peu plus tard - à partir de l'avant-dernière décennie avant $\mathbf{J}$.-C., à en croire A. Roth-Congès ${ }^{72}$ (ce qui s'accorde avec notre conception des moyens financiers dévolus à Nimes) - les arguments plaident en faveur d'une déduction antérieure à 25 .

Répétons-le : il ne s'agit que d'hypothèses. On aimerait les appuyer par un solide dossier onomastique. Or, si l'on note à Nimes la persistance de l'usage du grec, des liens avec des associations d'artistes grecs, l'existence de concours grecs, et même si ces

71 Ibidem, p. 123-129.

72 A. Rотн-Congt̀s, Le sanctuaire des eaux à Nimes, I. Le nymphée, Revue Archéologique du Centre, 22, 1983, étudiant le socle du "nymphée", propose (p. 135) l'avant-dernière décennie av. J.-C. pour la réalisation de sa frise, très imprégnée de modèles "gréco-asiatiques" et exécutée "par une équipe dont l'origine orientale ne parait pas douteuse" (p. 138). En revanche, les chapiteaux des colonnes libres de ce socle se rattacheraient à des modèles romains imprégnés du néoatticisme qui inspire l'Ara Pacis; il faudrait donc les dater du début de la dernière décennie avant J.-C. Conclusion (p. 142) : on doit admettre "une brève interruption dans le programme augustéen d'aménagement du sanctuaire et le renouvellement du personnel chargé de la décoration: l'ancien atelier est relayé par une équipe plus attentive aux nouvelles directives venues de Rome». Cette distinction correspondrait bien à la rupture que nous suggérons: une décision augustéenne vers 16-13 et un essor architectural à partir de ces mêmes décennies.

73 Les gentilices issus de noms grecs: Chrysius (ILGN, 452), Anthius (CIL, XII, 4188) et Scymnius (inédit). Une grande famille, celle des Solonii, est connue, surtout au II $\mathrm{s}$., par Q. Solonius Severinus $(C I L, \mathrm{XII}, 3184)$ : cf. Y. Burnand, Sénateurs ..., p. 779-782; elle est peu répandue ailleurs (XII, traits sont plus accusés que partout ailleurs en Narbonnaise, on ne relève que trois gentilices issus de noms grecs $^{73}$. Est-ce peu ou beaucoup? Il est difficile de trancher, car l'originalité de Nimes, à la différence des colonies romaines de vétérans, c'est sa faculté d'assimilation. Dans une communauté de droit latin, se sont fondus sans heurts des colons, eux aussi de droit latin, qui n'ont pas éliminé les grandes familles arécomiques : l'intégration d'une tour protohistorique dans la Tour Magne, point culminant de l'enceinte d'Auguste et dominant le "sebasteion", peut plaire à qui aime le symbole ${ }^{74}$. Pour lui adjoindre le concret, une déduction "gréco-orientale" à Nimes n'a rien d'absurde : aux alentours de 30 avant J.-C., on devait encore y comprendre le grec, et pour certains, le parler et l'écrire ${ }^{75}$.

\section{Michel Christol et Christian Goudineau}

3924, 2789, 3165 b). Pour le reste, mise au point de P. Gros, L'augusteum, p. 130-132.

74 P.-A. FÉvrier, Le souvenir de la petite patrie et l'histoire, La patrie gauloise d'Agrippa au vre siècle, Lyon, 1981, p. 19-25.

75 M. LeJec ne, Textes gallo-grecs, R.I.G. I, suppl. 45 à Gallia, 1985. Sur un ensemble de 281 documents "gallo-grecs", 224 concernent la Transalpine. Parmi eux, 64 proviennent du département du Gard, 4 de l'Hérault, soit 68 du territoire des Arécomiques (où sont oubliées les trouvailles de Lattes et de Nages).

Cet article a été rédigé avant la parution de l'étude de R. Turcan, Les religions orientales en Gaule Narbonnaise et dans la vallée du Rhòne, $A N R W$, II, 18, 1, p. 456-518. On lira particulièrement les pages 462 à 466 sur l'importance des cultes égyptiens à Nimes, considérée comme le "grand pôle égyptisant de la Gaule Narbonnaise".

N.B. - La figure 3 nous a été aimablement fournie par le Musée Calvet d'Avignon. Pour les tigures 1, 4 et 5 , à l'exception des extraits de l'Atlas des Monnaies gauloises de II. de La Tour, les photographies nous ont été données par J.-B. Giard, Conservateur au Cabinet des Médailles, que nous remercions vivement ici. 